\title{
Studies of deuteron and neutron cross-sections important for ADS research
}

\author{
V. Wagner ${ }^{1}$, J. Vrzalová, M. Suchopár, O. Svoboda, M. Majerle, A. Kugler \\ Nuclear Physics Institute ASCR \\ 25068 Řež, Czech Republic \\ E-mail:wagner@ujf.cas.cz, suchopar@ujf.cas.cz,vrzalova@ujf.cas.cz, \\ svoboda@ujf.cas.cz, majerle@ujf.cas.cz, kugler@ujf.cas.cz
}

\section{J. Adam, A. Baldin, W. Furman, M. Kadykov, A. Solnyshkin, V. Tsupko-Sitnikov, S. Tyutyunikov, N. Vladimirovna, L. Závorka}

Joint Institute of Nuclear Research

Joliot Curie str. 6 ,1419980 Dubna, Moscow region, Russia

E-mail: iadam@jinr.ru, Anton.Baldin@sunhe.jinr.ru, furman@jinr.ru, kadykov@jinr.ru, soln@sunhe.jinr.ru, vtsoupko@jinr.ru, tsi@sunhe.jinr.ru, vladimirovna@jinr.ru, zavorka@jinr.ru

\section{Bielewicz, S. Kilim, M. Szuta, E. Strugalska-Gola}

National Centre for Nuclear Research

04-500 Otwock-Swierk, Poland

E-mail:m.bielewicz@cyf.gov.pl,s.kilim@cyf.gov.pl,mszuta@cyf.gov.pl, elasg@cyf.gov.pl

\begin{abstract}
The collaboration Energy and Transmutation of Radioactive Waste uses different setups consisting of lead, natural uranium and graphite irradiated by relativistic protons and deuterons to study transmutation of radioactive materials by produced neutrons. The activation samples are used to determine integral of proton or deuteron beams and also produced neutron flux in different places of experimental set-ups. Unfortunately almost no experimental cross-section data for deuterons with $\mathrm{GeV}$ energies are available. The similar situation is also for threshold $(\mathrm{n}, \mathrm{xn})$ reactions of neutrons with higher energies. Therefore we carried out series of experiments devoted to determination of deuteron reactions on copper during uranium target QUINTA irradiations by deuterons with energies from $1 \mathrm{GeV}$ up to $8 \mathrm{GeV}$. The cross-sections of various threshold reactions were studied by means of different quasi-monoenergetic neutron sources with possible energies from $14 \mathrm{MeV}$ up to $100 \mathrm{MeV}$. Knowledge of such cross-sections is very important for all Accelerator Driven System studies.
\end{abstract}

XXI International Baldin Seminar on High Energy Physics Problems September 10-15, 2012

JINR, Dubna, Russia

1

Speaker 


\section{Introduction}

Activation detectors are commonly used to measure integral flux of different particles during Accelerator Driven System (ADS) studies. Determination of proton or deuteron beam integral intensity during ADS irradiation is possible by means of mainly the aluminum or copper foils. Activation samples make also possible to measure neutron flux in different places of ADS set-ups. The samples are small and their placement inside set-up is very simple. Approximate determination of neutron spectra is possible by use of different materials and different threshold reactions. Significant voids in the cross-section libraries of different important reactions are the main problem of effective work with the activation detectors. This gap was the main reason for us to start series of cross-section studies.

\section{Cross-sections of relativistic deuteron reactions on copper}

We participate in ADS studies performed by "Energy and Transmutation of Radioactive Waste" (E\&T RAW) international collaboration at JINR Dubna [1]. The accelerator Nuclotron is used as source of relativistic protons and deuterons for such studies. The aluminum and copper foils are used to determine beam integral during different experiments of our collaboration. Cross-sections of relativistic proton reactions with aluminum and copper are mostly known. Situation is very different for relativistic deuterons. Set of cross-sections for deuteron reaction ${ }^{27} \mathrm{Al}(\mathrm{d}, 3 \mathrm{p} 2 \mathrm{n}){ }^{24} \mathrm{Na}$ is very scare for energies higher than $200 \mathrm{MeV}$ and experimental values of cross-sections for deuteron reactions on copper are completely missing. This was the reason why we started program of measurements of these cross-sections.

The measurements were performed during irradiations of QUINTA and GAMMA-3 set-ups of E\&T RAW collaboration by means of deuteron beams from Nuclotron accelerator at JINR Dubna. Overall ten irradiations were performed during three sets of E\&T RAW collaboration experiments (March 2011, December 2011 and March 2012). The integral intensities of deuteron beams were determined by production of ${ }^{24} \mathrm{Na}$ in aluminum foil, for more detailed description see [2]. The common irradiation of aluminum and copper foils made possible to determine cross-sections of production of different radioisotopes by reactions of relativistic deuterons with copper. The used copper foils have natural isotope composition $\left(69.15 \%\right.$ of ${ }^{63} \mathrm{Cu}$ and $30.85 \%$ of ${ }^{65} \mathrm{Cu}$ ). Many radioisotopes with suitable decay time and gamma lines are produced during irradiation of copper by relativistic deuterons.

\subsection{Activation method}

Activation method was used to determine cross sections. Copper foil with size $10 \times 10 \mathrm{~cm}$ with thickness $0.0128 \mathrm{~cm}$ was placed together with aluminum foil with the same size and thickness $0.0196 \mathrm{~cm}$ several meters far away from the experimental set-up in the direction to beam tube window. Both foils were irradiated by same beam portion in the same place. The 
distance was sufficient to neglect possible influence of back-scattered neutrons and other particles from irradiated set-up.

The yield (i.e., the number of nuclei activated in the foil during the whole period of the irradiation) of produced gamma-radioactive nuclei was determined with the help of gammaspectroscopy. The foil was packed from original size to a smaller one with dimensions approximately $2.5 \times 2.5 \times 0.3 \mathrm{~cm}^{3}$ for the spectroscopy measurement. Activated foils were measured by two or more detectors and also in more different geometries which were in the range from $4 \mathrm{~cm}$ up to $10 \mathrm{~cm}$ far from detector. More measurements were done to see and identify short lived and long lived radioisotopes. We started with short measurements (minutes) and ended with long measurements (days).

The detectors were calibrated using standard laboratory ${ }^{54} \mathrm{Mn},{ }^{57} \mathrm{Co},{ }^{60} \mathrm{Co},{ }^{109} \mathrm{Cd},{ }^{133} \mathrm{Ba}$, ${ }^{137} \mathrm{Cs},{ }^{152} \mathrm{Eu},{ }^{228} \mathrm{Th}$, and ${ }^{241} \mathrm{Am}$ point sources which have several gamma-lines ranging from $80 \mathrm{keV}$ up to $2610 \mathrm{keV}$. Evaluated calibration activities include correction on real coincidences for isotopes with more lines. Total number of points used for one calibration curve is more than 35 .

Gamma-spectra were evaluated in the Deimos 32 code [3]. From the gauss-fit of the peaks we got also the statistical uncertainty. Total yield of ${ }^{24} \mathrm{Na}$ in the Al foil was determined according to the classic equation and all spectroscopic corrections were included, for details see [4]). The total uncertainty of the yield is gained by sum of statistical uncertainty, uncertainty of detector efficiency determination (mostly approximately $3 \%$ ) and different corrections uncertainties (mostly approximately $1 \%$ ).

The total beam intensity was determined by the activation analysis method using foil from ${ }^{27} \mathrm{Al}$. The knowledge of ${ }^{27} \mathrm{Al}(\mathrm{d}, 3 \mathrm{p} 2 \mathrm{n}){ }^{24} \mathrm{Na}$ reaction cross-section is crucial for determination of absolute normalization of obtained copper reaction data.

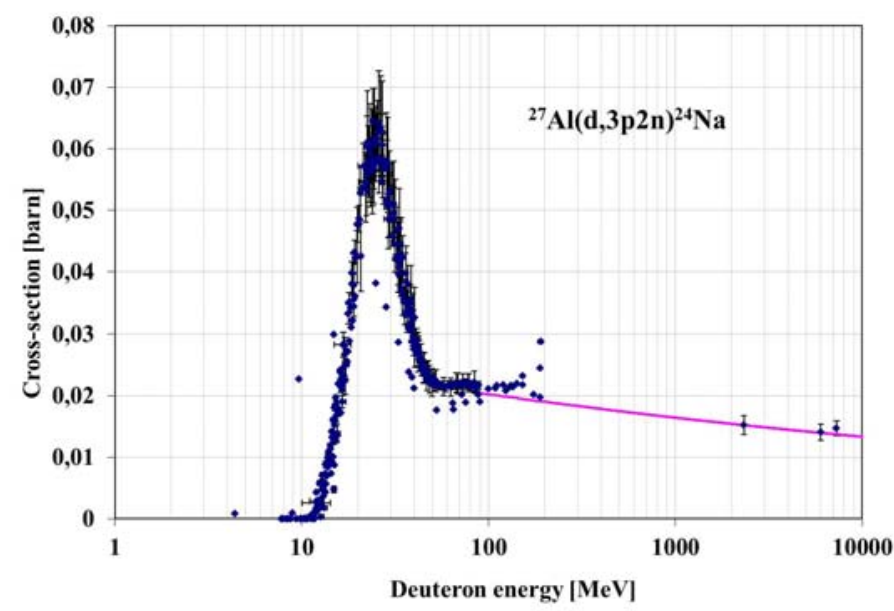

Fig. 1) Cross-section of ${ }^{27} \mathrm{Al}(\mathrm{d}, 3 \mathrm{p} 2 \mathrm{n})^{24} \mathrm{Na}$ reaction as function of energy - experimental data from EXFOR and fit of the points. 


\subsection{Cross-sections of ${ }^{27} \mathrm{Al}(\mathrm{d}, 3 \mathrm{p} 2 \mathrm{n}){ }^{24} \mathrm{Na}$ reaction}

Unfortunately, there are only three experimental cross-section values for ${ }^{27} \mathrm{Al}(\mathrm{d}, 3 \mathrm{p} 2 \mathrm{n}){ }^{24} \mathrm{Na}$ reaction in the $\mathrm{GeV}$ energy range. One value is from Dr. Bainaigs (15.25 \pm 1.5 mbarn at $2.33 \mathrm{GeV})[5]$ and two are from Dr. Kozma $(14.1 \pm 1.3$ mbarn at $6 \mathrm{GeV}$ and $14.7 \pm 1.2 \mathrm{mbarn}$ at $7.3 \mathrm{GeV})[6]$, see Fig. 1.

We made a fit by function $\sigma=\mathrm{a} \cdot \mathrm{E}^{\mathrm{b}}$ (linear in log scale of energy) between these three points and calculated the cross-section value for the two energies between the points from EXFOR database [7], we got a value of 16.4 mbarn for $1 \mathrm{GeV}$, a value of 14.5 mbarn for $4 \mathrm{GeV}$ and a value of 13.6 mbarn for $8 \mathrm{GeV}$. The error is about $10 \%$ and we use this uncertainty for the cross-section in our calculations.
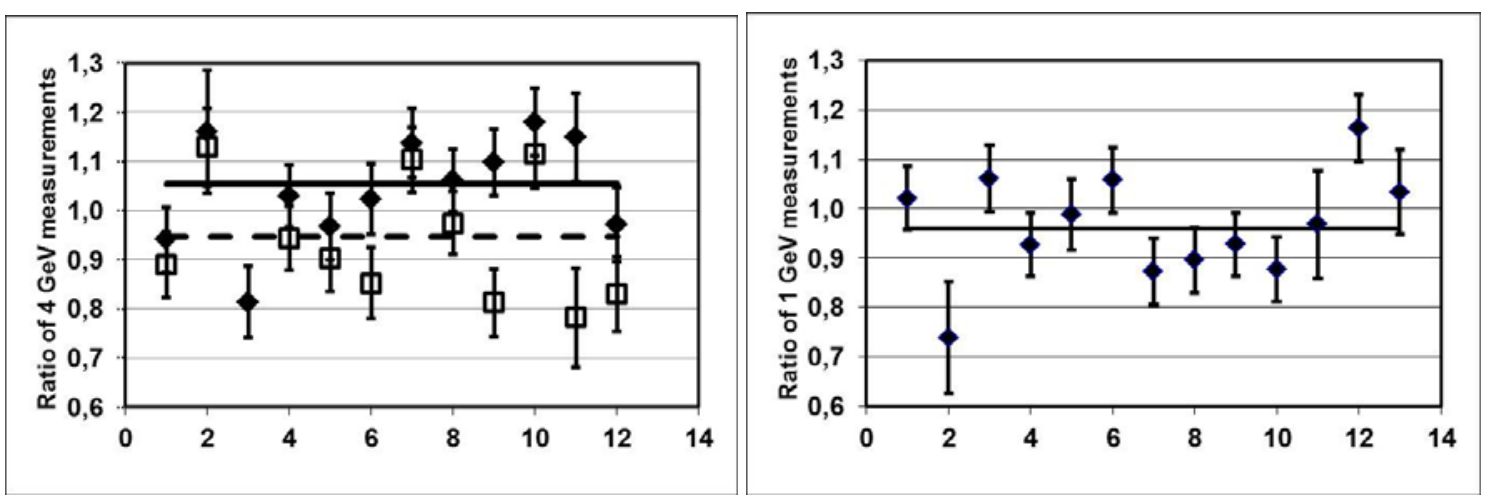

Fig. 2) Ratios of cross-sections obtained during three measurements with $4 \mathrm{GeV}$ deuteron beam (left). Order of isotopes is ${ }^{58} \mathrm{Co},{ }^{56} \mathrm{Co},{ }^{56} \mathrm{Mn},{ }^{52} \mathrm{Mn},{ }^{48} \mathrm{Sc},{ }^{44 \mathrm{~m}} \mathrm{Sc},{ }^{57} \mathrm{Ni},{ }^{48} \mathrm{~V},{ }^{47} \mathrm{Sc},{ }^{55} \mathrm{Co},{ }^{48} \mathrm{Cr}$ and ${ }^{43} \mathrm{~K}$. Ratio of March 2011 and March 2012 is shown by empty boxes (single ratios) and dashed line (mean weighted value). Ratio of December 2011 and March 2012 is shown by full triangles and full line. Ratios of two measurements with $1 \mathrm{GeV}$ beam (March 2012 / December 2011) are shown in the same manner in right figure.

\subsection{Cross-sections of different radioisotope production by deuteron copper reactions}

Gamma lines of the thirteen different radioisotopes $\left({ }^{43} \mathrm{~K},{ }^{43} \mathrm{Sc},{ }^{44 \mathrm{~m}} \mathrm{Sc},{ }^{47} \mathrm{Sc},{ }^{48} \mathrm{Sc},{ }^{48} \mathrm{~V},{ }^{48} \mathrm{Cr}\right.$, ${ }^{52} \mathrm{Mn},{ }^{56} \mathrm{Mn},{ }^{55} \mathrm{Co},{ }^{56} \mathrm{Co},{ }^{58} \mathrm{Co}+{ }^{58 \mathrm{~m}} \mathrm{Co}$ and ${ }^{57} \mathrm{Ni}$ ) were identified in the obtained gamma spectra. Radioisotope ${ }^{56} \mathrm{Co}$ has the shortest half-life (2.6 hours) among them and radioisotope ${ }^{58} \mathrm{Co}$ the longest one (1700.6 hours). Radioisotopes ${ }^{48} \mathrm{Sc}$ (43.7 hours) and ${ }^{48} \mathrm{~V}$ (383.4 hours) decay both to the same daughter nucleus ${ }^{48} \mathrm{Ti}$. The analysis of the $\mathrm{A}=48$ isobars is complicated also by the sequence of the decays ${ }^{48} \mathrm{Cr} \rightarrow{ }^{48} \mathrm{~V} \rightarrow{ }^{48} \mathrm{Ti}$.

Radioisotopes ${ }^{43} \mathrm{~K}$ (22.3 hours) and ${ }^{43} \mathrm{Sc}$ (3.9 hours) decay both to the same daughter nucleus ${ }^{43} \mathrm{Ca}$. The gamma line with energy $617.49 \mathrm{keV}$ is produced only by ${ }^{43} \mathrm{~K}$ decay and it is possible to use its intensity as norm to suppress contribution of ${ }^{43} \mathrm{~K}$ to gamma line with energy 
$372.8 \mathrm{keV}$ during first few hours after end of irradiation. We will obtain yield of ${ }^{43} \mathrm{Sc}$ decay using $372 \mathrm{keV}$ line after subtraction of ${ }^{43} \mathrm{~K}$ contribution.

Similar analysis was done for decays of radionuclides ${ }^{56} \mathrm{Mn}$ (2.6 hours) and ${ }^{56} \mathrm{Co}$ (1854.5 hours). The gamma line with energy $1238.3 \mathrm{keV}$ is produced only in ${ }^{56} \mathrm{Co}$ decay and it is possible to use it for calculation of contribution of ${ }^{56} \mathrm{Co}$ decay to $846.8 \mathrm{keV}$ line during first hours after end of irradiation. The yield of ${ }^{56} \mathrm{Mn}$ is determined after subtraction of calculated contribution of ${ }^{56} \mathrm{Co}$ decay.
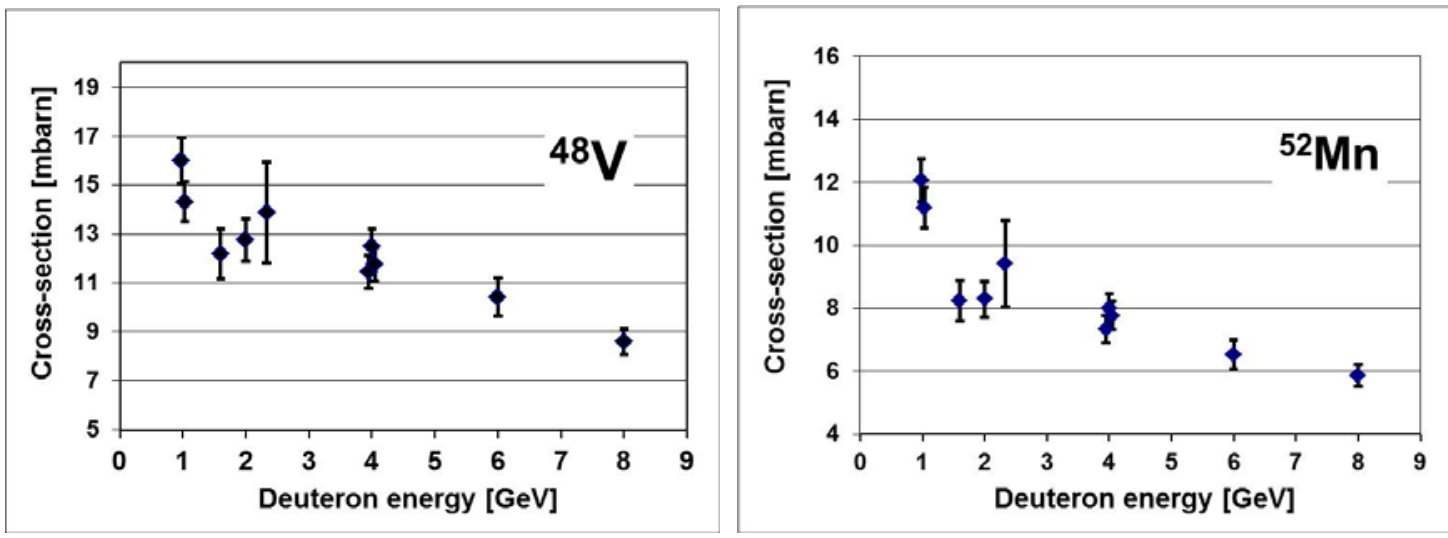

Fig. 3) Cross-sections of production of ${ }^{48} \mathrm{~V}$ and ${ }^{52} \mathrm{Mn}$ by deuteron reaction on natural copper are shown.
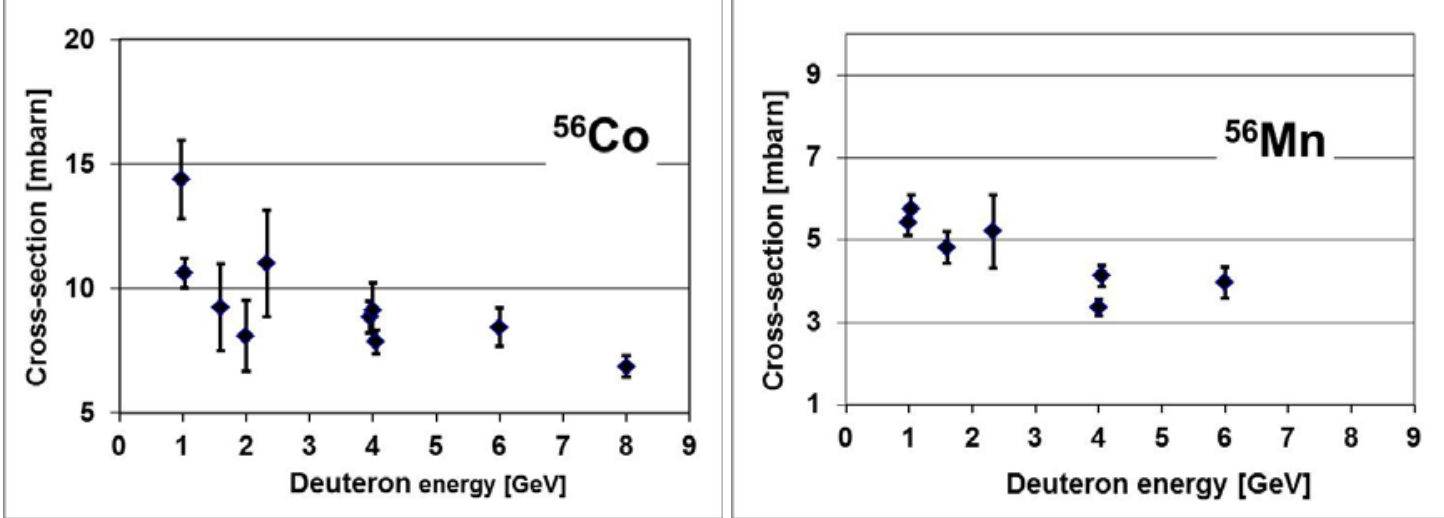

Fig. 4) Cross-sections of production of ${ }^{56} \mathrm{Co}$ and ${ }^{56} \mathrm{Mn}$ by deuteron reaction on natural copper are shown. These two radionuclides have the same daughter nucleus ${ }^{56} \mathrm{Fe}$.

The statistical uncertainties, uncertainties of efficiency, spectroscopic corrections and subtractions are different for individual lines (radionuclides). The uncertainty of beam integral determination is same for all radionuclides measured in the same irradiation. We made irradiation by the $4 \mathrm{GeV}$ deuteron beam three times and their systematic differences are within 
$10 \%$. Mean value of ratios between March 2011 and March 2012 results is 0.95(3) and mean value of ratios between December 2011 and March 2012 results is 1.05(2). Mean value of ratios between March 2012 and December 2011 results obtained during $1 \mathrm{GeV}$ beam is 0.96(2). More detailed information is shown in Fig.2. It is consistent with expected uncertainty of beam integral determination.

Excitation functions of thirteen different radioisotopes were determined within the range of energies from $1 \mathrm{GeV}$ up to $8 \mathrm{GeV}$. Several examples are shown in Fig. 3 and 4.

\section{Cross-sections of neutron reactions}

We routinely use threshold reactions in various materials to measure neutron flux during ADS studies. The usually used materials for neutron activation detectors are $\mathrm{Al}, \mathrm{Au}, \mathrm{Bi}, \mathrm{Co}, \mathrm{In}$, $\mathrm{Ta}$ and Y. Unfortunately almost no experimental cross-section data for observed threshold $(n, x n)$ reactions are available for higher neutron energies. Therefore we carried out series of experiments devoted to determination of neutron cross-sections of various threshold reactions using different quasi-monoenergetic neutron sources.

\subsection{Quasimonoenergetic neutron sources}

The quasi-monoenergetic neutron sources based on proton beam, lithium target and reaction ${ }^{7} \mathrm{Li}(\mathrm{p}, \mathrm{n})^{7} \mathrm{Be}$ are ideal tools for neutron reaction cross-section measurements. We used two such sources. The first neutron source is based on the cyclotron at Nuclear Physics Institute (NPI) in Ǩež with proton energy up to $38 \mathrm{MeV}$ [8]. Flux density up to $10^{9} \mathrm{~cm}^{-2} \mathrm{~s}^{-1}$ is possible. The second one is based on the cyclotron at The Svedberg Laboratory (TSL) in Uppsala with possible proton energy up to $200 \mathrm{MeV}$ [9]. Flux density around $10^{5} \mathrm{~cm}^{-2} \mathrm{~s}^{-1}$ is possible. We performed nine cross-section experiments exploiting the neutron source at NPI in Řež and seven irradiations by means of the neutron source at TSL in Uppsala using different energies of produced neutrons from 17 up to $94 \mathrm{MeV}$.

\subsection{Activation measurement of cross-sections}

Activation method was used for cross-section determination. The same technique as in previous case is applied in these experiments. Yield of observed isotopes (products of the ( $\mathrm{n}, \mathrm{xn})$ reactions) was calculated with respect to the various spectroscopic corrections - corrections on decay of the isotope between the end of irradiation and beginning of the measurement, correction on the intensity of the $\mathrm{I}_{\gamma}$ transition, correction on dead-time of the detector, correction on real $\gamma-\gamma$ cascade coincidence, self-absorption correction, square-emitter correction (geometrical correction), detector efficiency, and beam instability correction. The background yield was also calculated, subtracted and cross-section was calculated by means of formulae in articles $[11,12,13,14]$. 


\subsection{Examples of results}

Seven irradiations with energies 22, 47, 59, 66, 72, 89 and $94 \mathrm{MeV}$ were performed by means of quasi-monoenergetic neutron source at TSL in Uppsala. They were supplemented with irradiations on similar neutron source at NPI Řež with accurate neutron energies 17.5, 21.9, 30.4, 32.5 and $35.9 \mathrm{MeV}$. The $\mathrm{Au}, \mathrm{Al}, \mathrm{Bi}$, In and Ta materials were studied in all irradiations. The yttrium was studied during last two years. Cross-sections of threshold reactions ( $n, 2 n)$, $(n, 3 n)$ for these samples exist in EXFOR [7] up to $30 \mathrm{MeV}$, but no experimental data exist for higher energies or $(n, x n)$ reactions of higher order.

Comparison between the data from EXFOR, TALYS [15] and results from the measurements from Řež and Uppsala was made. Examples of the results can be seen in following graphs (Fig. 5 and Fig. 6). Good agreement with TALYS 1.4 and EXFOR data (where exist) is observed for most of the isotopes.
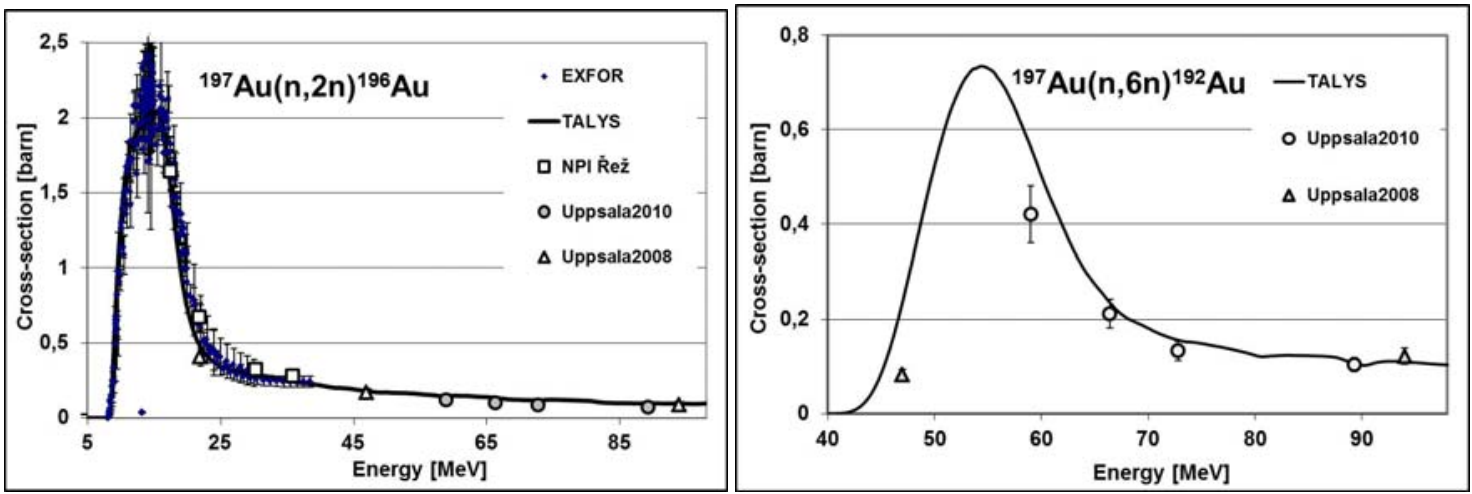

Fig. 5) Cross-sections of production of ${ }^{196} \mathrm{Au}$ and ${ }^{192} \mathrm{Au}$ by neutron reaction on gold are shown as examples of studied threshold reactions.
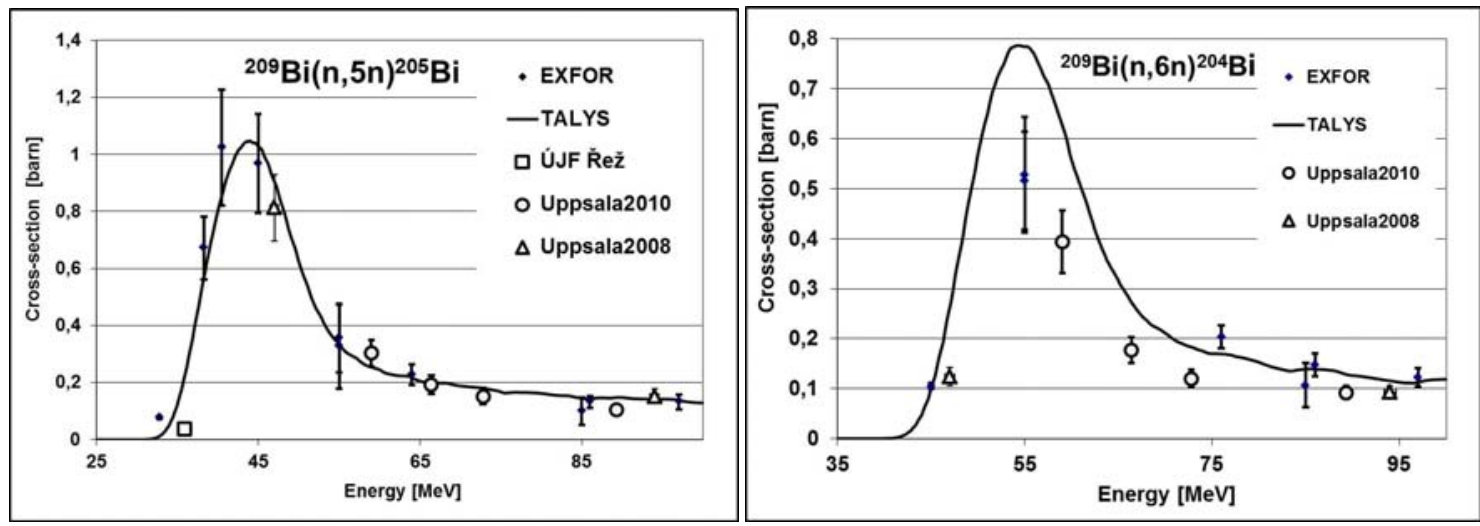

Fig. 6) Cross-sections of production of ${ }^{205} \mathrm{Bi}$ and ${ }^{204} \mathrm{Bi}$ by neutron reaction on bismuth are shown as examples of studied threshold reactions. 
We started detailed studies of neutron reactions with yttrium two years ago. The reaction ${ }^{89} \mathrm{Y}(\mathrm{n}, 3 \mathrm{n}){ }^{87} \mathrm{Y}$ is very interesting. Prior to their further decay, the nuclei are produced either in their ground state with half-life of 79.8 hours, or in the $380.79 \mathrm{keV}$ isomeric state with half-life 13.38 hours. The isomeric state decays by gamma transition to the ground state with probability $98.4 \%$. The beta decay of this state is within our accuracy negligible. We derived cross-sections of the ${ }^{89} \mathrm{Y}(\mathrm{n}, 3 \mathrm{n}){ }^{87} \mathrm{Y}$ and ${ }^{89} \mathrm{Y}(\mathrm{n}, 3 \mathrm{n}){ }^{87 \mathrm{~m}} \mathrm{Y}$ reactions from set of gamma spectra measured in different times. Results of first test of such analysis made by means of irradiation at NPI Řež are referred in [13]. Results of all previous irradiations at NPI Řež and TSL Uppsala are presented in Fig. 7. We already started new set of studies of neutron threshold reactions on yttrium.
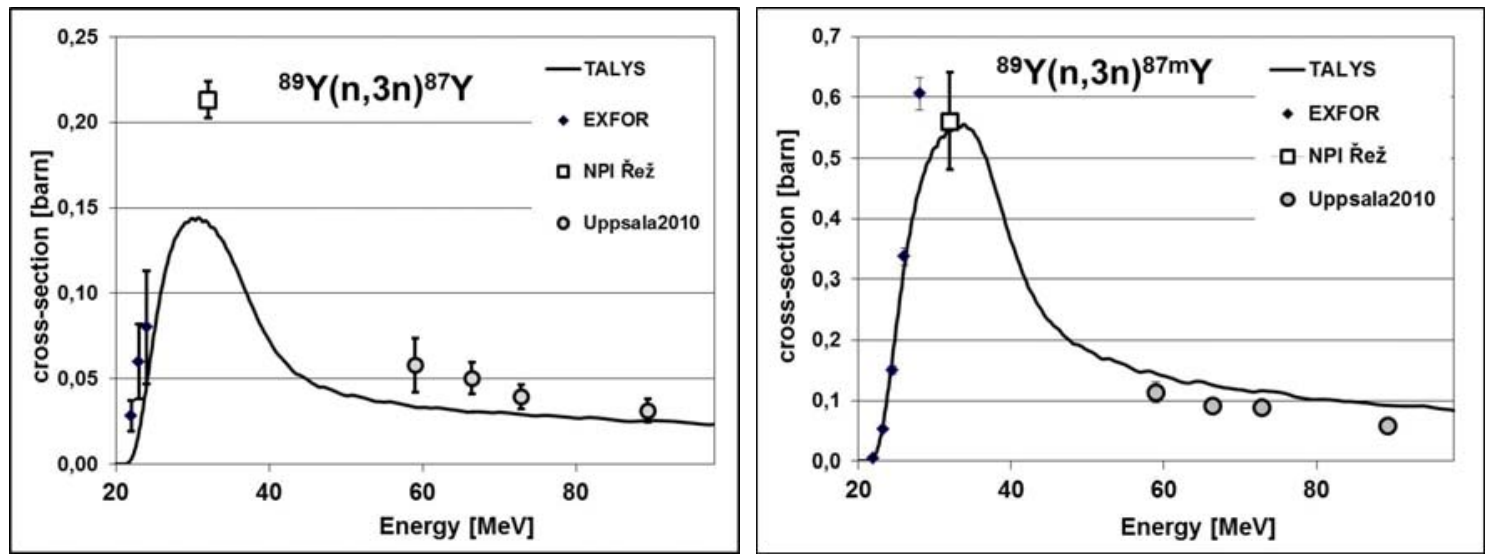

Fig 7) Studies of production of ${ }^{87} \mathrm{Y}$ and ${ }^{87 \mathrm{~m}} \mathrm{Y}$ by neutron reaction on yttrium were started.

\section{Conclusions}

The activation method was used to determine cross-sections of relativistic deuterons with natural copper. The cross-sections of thirteen different radioisotopes production $\left({ }^{43} \mathrm{~K},{ }^{43} \mathrm{Sc}\right.$, ${ }^{44 \mathrm{~m}} \mathrm{Sc},{ }^{47} \mathrm{Sc},{ }^{48} \mathrm{Sc},{ }^{48} \mathrm{~V},{ }^{48} \mathrm{Cr},{ }^{52} \mathrm{Mn},{ }^{56} \mathrm{Mn},{ }^{55} \mathrm{Co},{ }^{56} \mathrm{Co},{ }^{58} \mathrm{Co}$ and ${ }^{57} \mathrm{Ni}$ ) were determined within the deuteron energy range from $1 \mathrm{GeV}$ up to $8 \mathrm{GeV}$.

Original data on cross-sections of neutron threshold reactions were obtained for neutron energies above $17 \mathrm{MeV}$ by means of quasi-monoenergetic ${ }^{7} \mathrm{Li}(\mathrm{p}, \mathrm{n})^{7} \mathrm{Be}$ neutron sources at TSL Uppsala and NPI Řež. The results are in good agreement with the cross-sections already published in EXFOR. Good agreement is mostly with calculation by means of TALYS 1.0 code.

\section{Acknowledgements}

This work was supported by the Řež-Dubna funds, EFNUDAT program and grant number CTU0808214. The authors are grateful to the staff of the Dubna Nuclotron accelerator for providing a good deuteron beam and to the staff of TSL Uppsala and NPI Řež for neutron beams. We would also like to thank Pavel Bém, Eva Šimečková and Milan Honusek for the possibility of participation in their irradiations. 


\section{References}

[1] A. Krása et al., Neutron production in a Pb/U-setup irradiated with 0.7-2.5 GeV protons and deuterons, Nucl. Instr. and Meth. in Nucl. Res., A615 (2010) 70-77

[2] W. Furman at al., Recent Results on the Study of ADS with $500 \mathrm{~kg}$ Natural Uranium Target Assembly QUINTA Irradiated by Deuterons with Energies from 1 to $8 \mathrm{GeV}$ at JINR Nuclotron, this proceedings

[3] J. Frána, Program DEIMOS32 for Gamma-Ray Spectra Evaluation, J. Rad. Nucl. Chem., V. 257 , No. 3 P. (2003) 583-587.

[4] O. Svoboda, Experimental Study of Neutron Production and Transport for ADTT, Disertation Thesis, Czech Technical University, Faculty of Nuclear Sciences and Physical Engineering, Prague, 2011. [http://ojs.ujf.cas.cz/ wagner/transmutace/diplomky/PHD_Svoboda.pdf]

[5] J. Banaigs et al., Determination De L'Intensite D'Un Faisceau De Deutons Extrait D’Un Synchrotron Et Mesure Des Sections Efficaces Des Reactions C-12(D,P2N)C-11 Et Al27(D,3P2N)Na-24 a $2.33 \mathrm{GeV}$, Nuclear Instruments and Methods in Physics Research 95 (1971) 307-311

[6] P. Kozma and V. V. Yanovski, Application of $\mathrm{BaF}_{2}$ scintillator to off-line gamma ray spectroscopy, Czech Journal of Physics, 40 (1990) 393-397

[7] Experimental Nuclear Reaction Data (EXFOR/CSISRS) [http://www.nndc.bnl.gov/exfor], 7.4.2012

[8] P. Bém et al., The NPI cyclotron-based fast neutron facility, Proceedings of the International Conference on Nuclear Data for Science and Technology - ND2007, 555-558 (2007)

[9] A. V. Prokofiev et al., The TSL Neutron Beam Facility, Rad. Prot. Dos. 126 (2007) 18-22

[10] V. Wagner et al., Measurement of Neutrons in the Pb/U Assembly Irradiated by Relativistic Protons and Deuterons by means of Activation Samples, Proceedings of the XX International Baldin Seminar on High Energy Physics Problems, Dubna, October 2010, Vol. II, p. 253

[11] O. Svoboda et al., Proceedings of the International Conference on Nuclear Data for Science and Technology-ND2010, Jeju, South Korea, (2010) 1709-1712

[12] J. Vrzalová et al., Proceedings of the NEMEA-6 scientific workshop on Nuclear Measurements, Evaluations and Applications, Krakow, Poland, (2010) 259-265

[13] V. Wagner et al., Measurement of Neutrons in Different Pb/U Setups Irradiated by Relativistic Protons and Deuterons by means of Activation Samples, XIX Internatiol School on Nuclear Physics and Applications (VARNA 2011), Journal of Physics: Conference Series 366 (2012) 012047

[14] J. Vrzalová et al., Cross-section measurements of neutron threshold reactions in various materials, Physics Procedia 31(2012) 126-131

[15] A. J. Koning et al. TALYS 1.0. Proceedings of the International Conference on Nuclear Data for Science and Technology-ND 2007; 2007, 211-214. 